\title{
Influencia del liderazgo directivo en la satisfacción laboral docente
}

\section{Influence of directive leadership on teacher job satisfaction}

Wilson Rodas Hernández

Universidad César Vallejo, Perú

wrodas1975@gmail.com

(iD https://orcid.org/0000-0002-8583-5597

Manuel Ángel Pérez Azahuanche

Universidad César Vallejo, Perú

manuelangelperez@gmail.com

https://orcid.org/0000-0003-4829-6544

Recepción: 10/01/2021 | Aceptación: 21/04/2021 | Publicación: 10/05/2021

Cómo citar (APA, séptima edición):

Rodas Hernández, W., y Pérez Azahuanche, M. A. (2021). Influencia del liderazgo directivo en la satisfacción laboral docente. Innova Research Journal, 6(2), 90-104.

https://doi.org/10.33890/innova.v6.n2.2021.1684

\section{Resumen}

La persona que asume la conducción de una institución educativa vela por las metas, objetivos institucionales, tiene actitud inspiradora, es influyente y moviliza actividades de la comunidad educativa con acciones pedagógicas. Asimismo, los trabajadores demuestran satisfacción laboral al dar respuesta emocional efectiva referente al centro de trabajo, tienen seguridad laboral, se desarrollan profesionalmente y reconocen su trabajo con un salario; la finalidad del estudio fue determinar la influencia del liderazgo directivo en la satisfacción laboral en los docentes de la Institución Educativa $\mathrm{N}^{\circ}$ 80074,Virú 2020; la investigación fue no experimental de enfoque cuantitativo y diseño correlacional causal, se aplicaron dos cuestionarios de 25 y 27 ítems a la muestra de 88 participantes. Los resultados muestran 95\% Excelente Liderazgo directivo, el 63\% están satisfechos. Mediante el coeficiente de determinación en donde el 51,4\% tiene variabilidad de satisfacción laboral expresado por la influencia del Liderazgo directivo. Concluyendo que; se ejecutan acciones de liderazgo y se realiza actividades en conjunto, los docentes están a gusto con el trabajo que realizan, con el salario, tienen seguridad laboral y se desarrollan profesionalmente. Finalmente, este artículo contribuye en el aspecto científico al conocer que el liderazgo directivo influye en la satisfacción laboral docente.

Palabras claves: liderazgo directivo; satisfacción laboral; relación. 


\begin{abstract}
The person who assumes the leadership of an educational institution watches over the goals, institutional objectives, has an inspiring attitude, is influential and mobilizes activities of the educational community with pedagogical actions. Likewise, the workers show work satisfaction by giving an effective emotional response regarding the work center, they have work security, they develop professionally and recognize their work with a salary; the purpose of the study was to determine the influence of the management leadership on work satisfaction in the teachers of the Educational Institution $N^{\circ} 80074$, Virú 2020; the research was non-experimental of quantitative approach and causal correlation design, two questionnaires of 25 and 27 items were applied to the sample of 88 participants. The results show 95\% excellent management leadership, 63\% are satisfied. By means of the determination coefficient where $51.4 \%$ have variability of work satisfaction expressed by the influence of the managerial Leadership. Concluding that; leadership actions are executed and activities are carried out jointly, teachers are comfortable with the work they do, with the salary, they have job security and they develop professionally. Finally, this article contributes in the scientific aspect by knowing that the directive leadership influences in the teachers' labor satisfaction.
\end{abstract}

Keywords: executive leadership; job satisfaction; relationship.

\title{
Introducción
}

A nivel mundial que en una institución o empresa el funcionamiento de esta se debe a la existencia de un buen ejercicio de liderazgo de su director o gerente lo cual incide en la satisfacción laboral de los trabajadores, los cuales son el equipamiento fundamental en una institución.

Según manifiesta la Consejo Nacional de Ciencia, Tecnología e Innovación Tecnológica (UNESCO), reconoce que el liderazgo regidor en las instituciones se ha convertido en un tema muy estratégico según las políticas educativas. Como considera Leithwood et al. (2006); McKinsey y Company, (2007) constatando como un valor sobresaliente la influencia del liderazgo en la eficacia y en el mejoramiento de instituciones formadoras, siendo considerado "el segundo factor intra escolar" de trascendencia mayoritaria.

Se puede apreciar que a nivel de Latinoamérica se está considerando la importancia de elaborar ciertas políticas de estado que promocionen la labor eficaz y eficiente del liderazgo directivo en las escuelas de educación básica regular como también de superior. Se puede apreciar el empoderamiento de muchas acciones e iniciativas a favor de los directivos de instituciones públicas lo que debe ser considerada que se tomen con cierta credibilidad y veracidad para el buen surgimiento de una calidad educativa a favor del estudiante.

Según los datos dados a conocer por el Segundo Estudio Regional Comparativo y Explicativo (SERCE, OREALC-UNESCO), asimismo, los resultados existentes según el Programa para la Evaluación Internacional de Alumnos (PISA, OECD). Murillo (2012) consolida, según los datos de SERCE, que las particularidades primordiales que podrían tener los directores de escuelas básicas de la región: Se observa que su edad promedio es de 44 años y la mayoría son mujeres, en Uruguay o Argentina tienen más de 85\% directoras mujeres, en México o Ecuador ellas conforman menos de la mitad, siendo las edades promedio es de 53 años, en Chile tienen desde 39 años al igual que en Guatemala o Nicaragua. También se observa que una gran mayoría 
de directores tienen títulos universitarios como también grado universitario los que trabajan en la zona urbana, además el $20 \%$ de docente tienen estudios en postgrado. La mayoría tiene contrato indefinido en su puesto y en México, Brasil o Guatemala tienen otro trabajo, así también un tercio de administradores educativos tendría menos horas de dedicación a las de 30 horas. Tienen escasas atribuciones para admitir a estudiantes, en Cuba y Nicaragua los directores pueden contratar y despedir a docentes. A pesar del pago que les dan a los directores ellos tienen gran satisfacción por su labor a pesar del vínculo con estudiantes, padres de familia y docentes.

Cigna International Markets, (2020) junto a en Kantar, quien fue el pionero en presentar los estudios realizados por 'Cigna COVID-19 Global Impact', luego del análisis de como la pandemia afecta en cuanto a su satisfacción laboral de los empleados. Anualmente se realiza este tipo de investigación ' $360^{\circ}$ Well-Being Survey 2020', que Cigna lo está haciendo desde el 2014 investiga aspectos relevantes sobre salud y bienestar en diversos aspectos, referente a la física, familiar, social, financiero y laboral. En la publicación realizada para el 2020 se ha realizado con la contribución de 10204 encuestados de China, Hong Kong, Singapur, España, Tailandia, Emiratos Árabes Unidos, Reino Unido y Estados Unidos, realizando en dos tiempos en enero y abril lo que coincidió con la crisis del coronavirus.

El Portal Randstad (2020) hace mención que la satisfacción de los colaboradores respecto a su trabajo y repercute directamente en su calidad de vida, lo cual es importante detectar para una institución cuan satisfechos están con la forma de trabajo desde sus hogares para poder continuar desde casa, los directivos, los gerentes tiene que monitorear como están atravesando esta crisis mundial de la pandemia como les está afectando para poder fomentar algún tipo de solución frente a ello. Es por ello se debe contar con vías de comunicación eficientes para escucharlos y a través de ello poder atender a sus necesidades, en estas situaciones el contacto con las personas esta mediado por la tecnología lo cual permite la comunicación.

En este estudio referente a liderazgo directivo se han considera las teorías que lo fundamentan como las; Teoría de la personalidad de Cattell (1972), menciona que la personalidad se manifiesta cuando nos dice lo que una persona hará cuando se encuentre en una situación determinada, asimismo manifiesta que al existir diferencias de personalidad existen también en las conductas, las cuales son formadas por constructos internos a los que se llaman rasgos que, son los descriptores de la personalidad del individuo, otorgando al ser humano un equilibrio temporal para reaccionar de una manera determinada frente a una situación. (Cloninger, 2002, p. 205)

Teoría X, y la Teoría Y de Mc Gregor (1960), quien hace una comparación de estilos antagónicos de la forma de administrar o liderar una institución. En la primera teoría se basa a la tradicional, mecanicista la que busca seguridad y cubrir sus necesidades fisiológicas, tuvo su base en convicciones erradas al ver al hombre como un perezoso, apático por su propia naturaleza, no asume responsabilidades está pensando en que lo dirijan; en cambio en la teoría Y se basa en concepciones modernas sobre el comportamiento del hombre, se basa en ideas actuales con respecto a la naturaleza del ser humano, no muestra desagrado por el trabajo, siendo este una fuente de satisfacción y agrado, asumen actitudes de cambio y perseverancia frente a las experiencias laborales. (Lidstone, 2003, pp. 17,18). 
En el presente estudio se da a conocer las definiciones sobre liderazgo directivo según el Ministerio de educación (2014, p.14), a través del Marco del Buen Desempeño Directivo se le da una connotación pedagógica que administrativa. Dicho liderazgo refleja: una preocupación por el nivel de predominancia en mejorar el aprendizaje de los alumnos. Por tanto, la diligencia de tipo administrativo e institucional encumbra su rol en garantizar la calidad de la gestión pedagógica.

Bolívar (2010, p. 10), quien entiende por liderazgo, primordialmente, a la destreza de influir en un grupo de personas, las cuales puedan tomar acciones según las que se propusieron ejerciendo una acción. El atender a un grupo de personas para conducirlos en el accionar de estrategias, acciones para la mejora de sus funciones dentro de una institución o empresa en la cual se encuentran inmersos estas acciones repercuten para una mejora estratégica en las instituciones. Urquijo y Bonilla (2008, p. 217), señalan al respecto: "Las habilidades directivas se refieren al conocimiento que conlleva este puesto para lograr la integración funcional y capacidad de coordinación en los distintos niveles operativos y administrativos de la empresa para lograr el éxito. Chiavenato, (2009) con la finalidad de lograr las metas organizacionales, el líder deberá tener en cuenta buenas relaciones con las personas con las cuales labora, trabajar en conjunto de acuerdo a los objetivos propuestos por la organización. Gutiérrez, (2003), todo líder deberá inspirar a sus trabajadores de manera intelectual y los animará con sus ideas únicas e innovadoras para resolver problemas. Bernal, (2000), el liderazgo permite motivar los logros de su personal, animarlos a tener nuevas ideas y asumir responsabilidades, proporcionar diferentes estrategias para resolver problemas, facilitar las cosas en lugar de imponerlas. Salazar y Pinedo (2011), definen al liderazgo con acciones orientadoras "arte de guiar a los trabajadores de una institución educativa considerando una sola visión a través de la persuasión, el pensamiento, las sugerencias, la compasión, la voluntad y la capacidad administrativa para lograr logros". Thieme (2005), da a conocer estilos típicos de algunos trabajadores: Tratarlos según su personalidad, reconocer sus logros o realizar un buen trabajo, respaldar a los empleados, comunicarse con sus colaboradores permanentemente, promover un buen clima laboral e intercambiar experiencias con las generaciones.

Gil (2014), reconoce que el líder es alguien que influye en otros; sus acciones o palabras motivan a los miembros del equipo a trabajar juntos para lograr objetivos comunes. Collao (1997), indica que el líder es el orientador, guía y gerente. Es un tipo que se encuentra en la cabeza de la estructura y su articulador. Describe exactamente el método para lograr sus objetivos y determina la visión de futuro que desea lograr. El Ministerio de educación $(2014$, p.16) a través de la información en el Marco del Buen Desempeño Directivo, refiere que, el liderazgo educativo, no es solamente tener en cuenta la calidad de la persona que dirige, debe dar ejemplo de liderazgo en la escuela, donde los miembros contribuyen al éxito de los resultados educativos. Longo (2008), menciona que, El liderazgo ejecutivo está íntimamente ligado al liderazgo equitativo, pues también busca que las organizaciones y grupos sociales tienden a ser una constelación de múltiples líderes, cuya vitalidad está relacionada en gran medida con el ejercicio de la descentralización y aceptación de decisiones. Ander-Egg, (1997), confirma que, el liderazgo es el papel que juega el líder en el equipo en determinados eventos carismáticos. El liderazgo tiene diferentes aspectos según la situación y las tareas: una persona puede ser líder de un grupo y sucesor de otro. La influencia se puede ejercer en áreas definidas o usarse para realizar tareas sin desempeñar un papel de liderazgo en otros entornos de desempeño. 
Fischman (2000), da a conocer las dimensiones, el Control del ego se utiliza para analizar los comportamientos de mando relacionados con el aumento del orgullo y la autoestima y los patrones de desfiguración; el Desapego, es la actitud de reclusión es "mantenerse alejado de las cosas, comprender el ciclo humano de la existencia y darse cuenta de que los problemas son una parte interna de la vida humana y pueden resolverse allí". La Responsabilidad, la gente suele pensar que no podemos hacer nada porque estamos prohibidos y estamos sin ningún accionar, es decir, encontramos algunas excusas para no aceptar nuestra responsabilidad. Trabajo en equipo: Empresas de todo el mundo invierten muchos recursos en la formación de sus equipos de trabajo, pero estos integrantes deben intentar desarrollar habilidades de liderazgo personal, de lo contrario esto se convierte en un obstáculo para el trabajo en equipo. Destrezas gerenciales, las personas en las organizaciones líderes deben tener muchas habilidades humanas.

Referente a Satisfacción laboral, se tuvo en cuenta a las siguientes teorías, según Maslow (1943), la teoría de Maslow conocida como la jerarquía de las necesidades humanas; al satisfacer las necesidades primordiales, existe un incremento en las necesidades y deseos complejos. Son cinco los niveles que constituyen la pirámide de Maslow; las primeras necesidad son de deficiencia las de la primera a la cuarta, y las necesidades de autorrealización se encuentran en el nivel cinco consideradas como las de motivación de crecimiento o necesidad de ser. Si se satisface las primeras en la necesidad del ser existe una fuerza impelente y continua. Al ser satisfechas las necesidades inferiores de la pirámide entonces tendremos más atención en las necesidades superiores.

La teoría de los factores de Herzberg (1959), reveló que el descontento y la satisfacción laboral se manifiestan de dos grupos independientes de factores. Considerados como los de higiene o mantenimiento y los de satisfacción o motivación, entre los de higiene están el sueldo y los beneficios, la política en la empresa o en la organización, las relaciones con los compañeros de trabajo, el ambiente físico, la supervisión, la seguridad laboral, el crecimiento, estos dan un mayor o menor grado de satisfacción al trabajador; y los factores de motivación son los logros, el reconocimiento, la independencia laboral, la responsabilidad, la promoción y la consolidación si estos no existieran durante la realización de su trabajo existe insatisfacción.

El modelo de expectativas de Vroom, el cual se basó en tres aspectos fundamentales: el primero referente a que el ser humano tiene un deseo interno por lograr sus objetivos que se traza, considerados como expectativas a futuro, el segundo aspecto se refiere como el individuo asocia su esfuerzo por su trabajo al lograr sus objetivos y por lo cual recibe una recompensa. Y el último aspecto es referente a la motivación que impulsan al individuo a considerarse como productivo al ser logrados sus objetivos este último es la consolidación de las expectativas como las recompensas.

Se consideran las siguientes definiciones de la variable satisfacción laboral, Muñoz (1990, p.176), refiere al hecho de que el sujeto se encuentra en el marco de una empresa u organización atractiva, en un entorno que te hace sentir cómodo y el hecho de que estás comprometido en un trabajo que te interesa y que es agradable o positivo. Realiza una serie de compensaciones psicológicas, sociales y económicas según sus expectativas. Días (2015, p.21), menciona que es un estado emocional, sentimiento o respuesta emocional relacionada con el trabajo. Presenta en el trabajo los conocimientos, la afectividad, y el comportamiento de los trabajadores frente al 
accionar de la ejecución en el trabajo. Zakaria y Mardela (2019), los maestros son muy importantes para la comunidad y nación, especialmente en colegio. Ayudan a los estudiantes con total responsabilidad de asegurarse de que ellos obtienen un buen resultado en sus evaluaciones.

Cantón y Téllez (2016) se tiene que considerar; actitud, sentimiento, emoción las que se relacionan con el clima, la salud mental y el equilibrio personal. Stoner (2006), manifiesta que la satisfacción laboral representa la preparación mental de los empleados antes de realizar el trabajo. Los trabajadores deben estar con su estado emocional en condiciones favorables para poder laborar. Manosalvas (2015), disposición del trabajador en la ejecución de sus laborares de acuerdo a sus funciones, que luego de realizarlo sientan satisfacción de haberlo logrado teniendo en cuenta la diversidad de factores para poder realizarlo los cuales contribuyen a ser exacto y preciso en la ejecución de su labor. Hannoun (2011), considera que una persona siente satisfacción por el trabajo realizado con tiene disposición de ejecutarlo con buen estado de ánimo con buena disposición, con empeño y esfuerzo realiza lo que se encomendó para sentirse bien consigo mismo y con la empresa. Inga (2016), manifiesta que la satisfacción laboral se observa en el trabajador cuando realiza su labor considerando un índice óptimo propuesto para una mejor productividad de la empresa, tiene seguridad en realizar lo que está pendiente, ejecutando con optimismo para lograr la meta propuesta.

También Zurita (2015), quien pone de conocimiento que los trabajadores que se sienten insatisfechos por la labor que realizan son factores de riesgo y es una amenaza para la empresa. Calvacante (2004), cuando hay personas que están encargados en la gestión institucional, en los subordinados se ven variaciones en cuanto a la labor a realizar y también y la calidad del trabajo se puede ver afectada y no tener un buen producto o lograr las metas propuestas. Gibson et al., (1993) con buena satisfacción laboral por parte de los trabajadores se puede lograr de manera muy favorables una calidad en la labor desempeñada. Amaya y Suárez (2004), manifiestan que la satisfacción laboral es evocada un buen estado emocional dando respuestas positivas en mejora del trabajo en lo cual habrá un mejor desempeño y un desenvolvimiento muy favorable en su accionar. Robbins (2006), encierra a actividades generales que las personas deben realizar de manera asertiva y pueda de esa manera demostrar actitudes positivas a favor del trabajo en el cual se desenvuelve.

Udyaningsih, Dwihartoa y Tahajjudi (2020), la satisfacción laboral es también un factor importante que influye en el desempeño de los empleados. Ser y estar capaces de mantener los recursos existentes, las empresas deben aumentar la satisfacción de los empleados, aumentar su compromiso organizacional y proporcionar seguridad laboral.

Palma (2006), tomo en cuenta las dimensiones referentes a: Significación de la Tarea, es prepararse para el trabajo y utilizar asignaciones relacionadas con el trabajo personal, refuerzo, perseverancia y / o aportes materiales. De la misma manera, se debe entender por condiciones de trabajo la valoración del trabajo realizado y la disponibilidad de los elementos, recursos o competencias que rigen el trabajo.

También es el reconocimiento personal y/o social, que se basa en las características del trabajo o en la valoración de la tendencia laboral por parte de la inspección de cooperación colectiva, y se refiere a los resultados obtenidos en el trabajo que tienen efectos o consecuencias 
indirectas. Asimismo, los beneficios económicos se refieren a la resolución del trabajo en lo que se refiere a los aspectos de pago, recompensa o compensación económica, como el interés en el ánimo y la valentía para realizar las tareas que se le encomiendan.

Ante lo descrito se plantea la pregunta de investigación: ¿Cómo el Liderazgo directivo influye en la satisfacción laboral en los docentes de la Institución Educativa N 80074, Virú 2020 ?

Para fundamentar la investigación, se tomó como antecedentes a los trabajos realizados por: Cantón y Téllez (2016), en la investigación; La satisfacción laboral y profesional de los profesores, en donde la mayoría de las damas de los niveles no universitarios tiene una satisfacción media-alta respecto a su carrera, recalcando que en el aspecto relación fue positiva, y también fue negativa en la valoración por su desempeño y sueldo. Montañez (2019), el estudio; Liderazgo directivo, motivación y desempeño docente en las instituciones educativas del nivel secundario del distrito de Platería-Puno, los resultados muestran correlación de 0,61, moderada positiva entre las variables en estudio y significativa, aceptando la hipótesis del investigador, que si existe relación significativa entre la primera variable y la segunda. Tovar (2018), en el estudio; Influencia del liderazgo del director en la satisfacción del alumno del diplomado en liderazgo y gestión del Grupo de Artillería de la Escuela de Artillería del Ejército - 2017. Concluye que el liderazgo del director influye significativamente en la satisfacción del estudiante en el desenvolvimiento de su aprendizaje. Cuya (2020), en el estudio; Liderazgo del director y satisfacción académica de los oficiales alumnos del diplomado en Liderazgo y Gestión del Grupo de Artillería, de la Escuela de Artillería del Ejército - 2018, concluyó que el ejercicio del liderazgo directivo tiene relación significativamente con la satisfacción académica de los oficiales, los cuales son estudiantes de un diplomado en liderazgo y gestión del grupo de artillería.

En el presente estudio se planteó el objetivo general: determinar la influencia del Liderazgo directivo en la Satisfacción laboral en docentes de la Institución Educativa № 80074, Virú 2020 y como objetivos específicos; Identificar la influencia del Liderazgo directivo en la dimensión Significación de la tarea de la Satisfacción laboral en docentes de la Institución Educativa N 80074, Virú 2020; Identificar la influencia del Liderazgo directivo en la dimensión Condiciones de trabajo de la Satisfacción laboral en docentes de la Institución Educativa $\mathrm{N}^{\circ}$ 80074, Virú 2020; Identificar la influencia del Liderazgo directivo en la dimensión Reconocimiento personal y/o social, de la Satisfacción laboral en docentes de la Institución Educativa N $^{\circ}$ 80074, Virú 2020; Identificar la influencia del Liderazgo directivo en la dimensión Beneficios económicos, de Satisfacción laboral en los docentes de la Institución Educativa $\mathrm{N}^{\circ}$ 80074, Virú 2020 .

\section{Metodología}

De acuerdo al planteamiento realizado para la presente investigación por su enfoque, naturaleza y también el carácter de medida es cuantitativo de tipo no experimental, es explicativa y según Hernández et al, (2010, p. 02) mencionan que: para la comprobación de las hipótesis utiliza los datos, los cuales tienen medición numérica, y están regidos de acuerdo a teorías. 
El diseño de investigación de acuerdo las características de las variables, es correlacional causal, de corte transversal en donde se observará por única vez si la primera variable influye en la segunda variable en un grupo de docentes a los cuales se les aplicó instrumentos previamente validados en otro contexto, determinando el grado de correlación causal en este contexto particular de una institución educativa.

La muestra lo conformaron 45 docentes correspondiendo al nivel primario y por 43 del nivel secundario en total 88 docentes.

Como medio de recolectar los datos se utilizó el cuestionario, cuyas preguntas permitirán la opinión sobre Liderazgo directivo, estuvo constituido por 25 ítems distribuidos en la dimensión Control del ego, Desapego, Responsabilidad, Trabajo en equipo, y Destrezas gerenciales cada una tiene 5 ítems, cuyos criterios fueron totalmente en desacuerdo 1 punto en desacuerdo 2 puntos, No sabe/no opina 3 punto, de acuerdo 4 puntos y totalmente de acuerdo 5 puntos.

Para la variable Satisfacción Laboral, el cuestionario lo conformó 27 ítems en Significación de la tarea 8 ítems, Condiciones de trabajo 9 ítems, Reconocimiento personal y/o social 5 ítems, y Beneficios económicos 5 ítems, teniendo como criterios Total desacuerdo 1 punto, en desacuerdo 2 puntos, indeciso 3 puntos, de cuerdo 4 puntos y totalmente de acuerdo 5 puntos. Se aplicaron los instrumentos en una sola vez a cada docente de la muestra.

\section{Resultados}

Se presentan los resultados obtenidos referente a las variables Liderazgo directivo y Satisfacción laboral recolectados por los instrumentos, datos alcanzados por los docentes del nivel primario y secundario.

\section{Tabla 1}

Prueba de normalidad para la variable Liderazgo directivo

\begin{tabular}{|c|c|c|c|}
\hline & \multicolumn{3}{|c|}{ Pruebas de normalidad } \\
\hline & \multicolumn{3}{|c|}{ Kolmogorov-Smirnov ${ }^{\mathrm{a}}$} \\
\hline & Estadístico & $\mathrm{gl}$ & Sig. \\
\hline Liderazgo directivo & ,078 & 88 & $200^{*}$ \\
\hline
\end{tabular}

Nota. Según datos obtenidos de la matriz.

$\mathrm{Al}$ realizar la prueba de contraste de la normalidad mediante Kolmogorov-Smirnov, se observa que, para Liderazgo directivo, el grado de significancia para la variable es mayor a 0,05, por lo cual se asume que los datos tienen una distribución normal. 
Tabla 2

Prueba de normalidad para la variable Satisfacción laboral

\section{Pruebas de normalidad}

\begin{tabular}{lrrr|r}
\hline & \multicolumn{3}{c}{ Kolmogorov-Smirnov $^{\mathrm{a}}$} & \multicolumn{2}{c}{ Sig. } \\
\hline Satisfacción laboral & Estadístico & gl & , \\
\hline
\end{tabular}

Nota. Según datos obtenidos de la matriz.

Al ejecutar la prueba de contraste de la normalidad mediante Kolmogorov-Smirnov, se observa que, para Satisfacción laboral, el grado de significancia para la variable es mayor a 0,05, por lo cual se asume que los datos tienen una distribución normal.

\section{Tabla 3}

Resultados de la variable Liderazgo directivo de docentes de la Institución Educativa $N^{\circ} 80074$, Viru 2020

\begin{tabular}{ccc}
\hline Niveles & f & \% \\
\hline Excelente & 84 & 95 \\
Bueno & 4 & 5 \\
Regular & 0 & 0 \\
Deficiente & 0 & 0 \\
\hline Total & 88 & 100 \\
\hline
\end{tabular}

Nota. Datos obtenidos de la matriz; $\mathrm{f}=$ Frecuencia absoluta; $\%=$ Frecuencia relativa.

Los docentes consideran al liderazgo directivo como Excelente en un 95\% la conducción de una institución lo realiza un líder que tiene bien claro las metas y objetivos.

\section{Tabla 4}

Resultados de la variable Satisfacción laboral de docentes de la Institución Educativa $N^{\circ} 80074$, Viru 2020

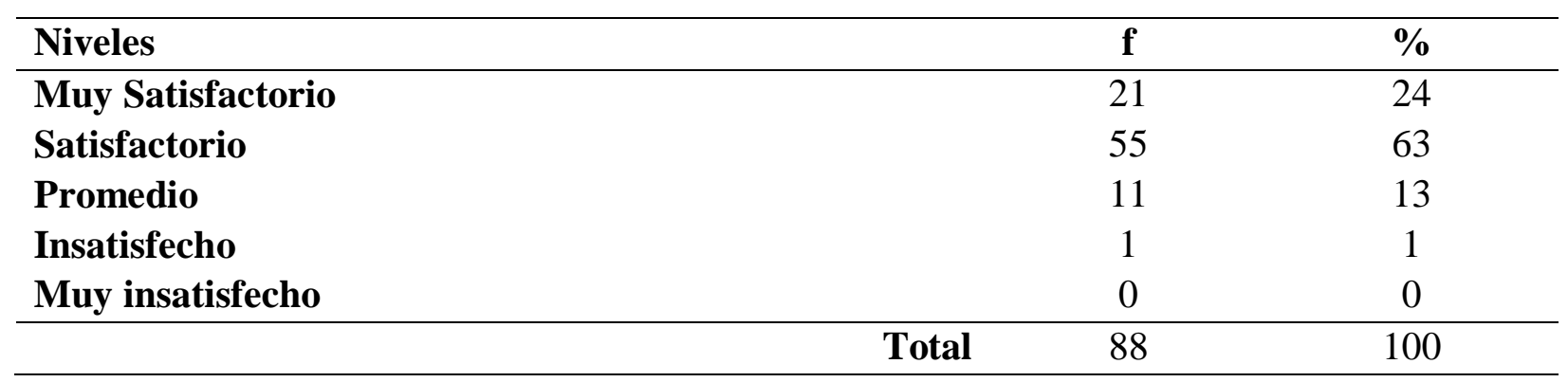


Nota. Datos obtenidos de la matriz; $\mathrm{f}=$ Frecuencia absoluta; $\%=$ Frecuencia relativa.

El 63\% consideran tener una Satisfacción laboral satisfactoria, dando a conocer una respuesta emocional efectiva al ejecutar acciones en su centro de trabajo, de acuerdo a sus obligaciones y condiciones de trabajo.

Dando respuesta estadísticamente al problema planteado referente sí, El liderazgo directivo influye significativamente en la satisfacción laboral de los docentes de la Institución Educativa $\mathrm{N}^{\circ}$ 80074, Virú 2020. Siendo necesario considerar de acuerdo a la normalidad de los datos y al tipo de escala de las variables al coeficiente de determinación, especificando los datos en las tablas siguientes:

\section{Tabla 5}

Coeficientes de Determinación R2

\begin{tabular}{|c|c|c|c|c|c|c|c|c|c|}
\hline \multirow[b]{2}{*}{ Modelo } & \multirow[b]{2}{*}{$\mathrm{R}$} & \multirow[b]{2}{*}{$\begin{array}{c}\mathrm{R} \\
\text { cuadrado }\end{array}$} & \multirow[b]{2}{*}{$\begin{array}{c}\mathrm{R} \\
\text { cuadrado } \\
\text { ajustado }\end{array}$} & \multirow{2}{*}{$\begin{array}{c}\text { Error } \\
\text { estándar } \\
\text { de la } \\
\text { estimación }\end{array}$} & \multicolumn{5}{|c|}{ Estadísticos de cambio } \\
\hline & & & & & $\begin{array}{c}\text { Cambio } \\
\text { en R } \\
\text { cuadrado }\end{array}$ & $\begin{array}{c}\text { Cambio } \\
\text { en F }\end{array}$ & gl1 & $\mathrm{g} 12$ & $\begin{array}{c}\text { Sig. } \\
\text { Cambio } \\
\text { en F }\end{array}$ \\
\hline 1 &, $717^{\mathrm{a}}$ &, 514 &, 508 & 5,942 &, 514 & 90,910 & 1 & 86 &, 000 \\
\hline
\end{tabular}

a. Predictores: (Constante), Liderazgo directivo

Nota. Datos obtenidos de la matriz; N= Muestra.

Se muestra en la tabla 5, la relación que existe entre las variables que, según el coeficiente de determinación es de 51,4\% de la variabilidad de satisfacción laboral la que está expresada por la influencia del Liderazgo directivo. También se observa el coeficiente de correlación de Pearson que es de 0,717 observándose que existe un alto grado de correlación positiva entre la variable independiente y la dependiente.

\section{Tabla 6}

\section{Análisis de Varianza ANOVA}

\begin{tabular}{llrrrrr}
\hline \multicolumn{2}{l}{} & \multicolumn{2}{c}{$\begin{array}{c}\text { Suma de } \\
\text { Modelo }\end{array}$} & \multicolumn{5}{c}{$\begin{array}{c}\text { Media } \\
\text { cuadrados }\end{array}$} & gl & & cuadrática & \multicolumn{1}{c}{ F } & \multicolumn{1}{c}{ Sig. } \\
\hline 1 & Regresión & 3209,620 & 1 & 3209,620 & 90,910 & ,001 $^{\text {b }}$ \\
& Residuo & 3036,278 & 86 & 35,306 & & \\
& Total & 6245,898 & 87 & & & \\
\hline
\end{tabular}

a. Variable dependiente: Satisfacción laboral b. Predictores: (Constante), Liderazgo directivo Nota. Datos obtenidos de la matriz; N= Muestra.

Según lo mostrado en la tabla 6, de ANOVA, el valor estadístico F es de 90,9 el que permite contrastar la hipótesis nula, observando una significancia menor a 0,05 lo que significa que, ambas 
variables están linealmente relacionadas. Esto indica la influencia significativa que tiene el liderazgo directivo en la satisfacción laboral según lo expresado por los docentes.

\section{Tabla 7}

Coeficientes de Regresión lineal para la hipótesis general

\begin{tabular}{|c|c|c|c|c|c|c|}
\hline & & \multicolumn{2}{|c|}{ Coeficientes no estandarizados } & \multirow{2}{*}{\begin{tabular}{|c|} 
Coeficientes \\
estandarizados \\
Beta \\
\end{tabular}} & \multirow[b]{2}{*}{$\mathrm{t}$} & \multirow[b]{2}{*}{ Sig. } \\
\hline \multicolumn{2}{|c|}{ Modelo } & $\mathrm{B}$ & Error estándar & & & \\
\hline \multirow[t]{2}{*}{1} & (Constante) & 28,076 & 8,671 & & 3,238 &, 002 \\
\hline & $\begin{array}{l}\text { Liderazgo } \\
\text { directivo }\end{array}$ &, 782 & ,082 &, 717 & 9,535 &, 000 \\
\hline
\end{tabular}

a. Variable dependiente: Satisfacción laboral

Nota. Datos obtenidos de la matriz; N= Muestra.

En la tabla 7 se observa el coeficiente de la constante el cual es el origen de la recta de regresión es de 28,076 siendo el punto intercepto con en el eje Y, también se observa que el coeficiente correspondiente a Liderazgo directivo es la pendiente de la recta de regresión el cual es igual a 0,782 , este valor indica que es positivo y permite determinar que existe una relación directa entre las variables. Según los resultados la ecuación sería: Satisfacción laboral = 0,782 Liderazgo directivo validando que existe una relación directa y positiva entre la satisfacción laboral y Liderazgo directivo.

\section{Discusión}

Posteriormente a la obtención de los resultados se realiza el análisis y la comparación de los mismos con los antecedentes y teorías, dando respuesta a los objetivos propuestos. Se observó que consideran un Excelente liderazgo directivo un 95\%, la conducción de la institución lo realiza un líder que tiene claro metas y objetivos, influye, inspira, y realiza las actividades de acuerdo a la totalidad de trabajadores; concuerda con el estudio realizado por Tovar (2018), manifiesta que el $84 \%$ de estudiantes consideran la realización de un Liderazgo positivo el cual es influyente en la satisfacción laboral de los docentes. Reafirmando que hay un avance en el Liderazgo ejercido por la persona que lo conduce y coordina con todos los trabajadores para sacar adelante la ejecución de los objetivos y lograr las metas institucionales. También Garay (2016), refiere que el Liderazgo es necesario para comprender efectos que ocurren dentro de las organizaciones escolares, por lo cual este manejo de liderazgo ayuda a que puedan salir adelante.

La influencia de la primera variable hacia la segunda según el coeficiente de determinación es de 51,4\% de la variabilidad de satisfacción laboral la que está expresada por el influjo del Liderazgo directivo. También se observa el coeficiente de correlación de Pearson que es de 0,717 observándose que existe un alto grado de correlación positiva entre la variable independiente y la 
dependiente. Para corroborar Machaca (2019), encontró una correlación positiva y muy significativa según Pearson de $0.568 * *$ entre el liderazgo directivo y la satisfacción laboral y con nivel de error del 0.05 o 5\% según los docentes de las instituciones secundarias públicas de la ciudad de Puno. Lo que permite afirmar que si existe relación y es relevante y si existe relación considerable que avala un buen sustento a la presente investigación.

Leithwood (2009), menciona el liderazgo es una labor que moviliza, influye en otros para articular y logra intenciones y metas a compartidas en una institución educativa lo cual hace que exista una influencia del liderazgo en acciones a ejecutar en los docentes. Este aporte favorece la intención que debe tener el líder hacia los colaboradores y poder fortalecer la tarea que realizan los docentes en la institución educativa.

Palma (2006), refiere que los beneficios económicos el cual tiene que ver con el salario que recibe al estar a cargo de una institución el cual va de acorde con las funciones que realiza, teniendo algún salario adicional como todo trabajador respecto a la labor que ejecuta. Un buen líder realiza sus actividades con responsabilidad y en algunas veces se considera que será reconocido por las personas que lo conocen o por las instituciones estatales que valoraran la labor.

La Teoría X, y la Teoría Y de Mc Gregor (1960), quien hace una comparación de estilos antagónicos de la forma de administrar o liderar una institución. En la primera teoría se basa a la tradicional, mecanicista la que busca seguridad y cubrir sus necesidades fisiológicas, en cambio en la teoría Y se basa en concepciones modernas sobre el comportamiento del hombre, no muestra desagrado por el trabajo. (Lidstone, 2003, pp. 17,18)

La teoría de los factores de Herzberg (1959), al referir la existencia de dos tipos de factores que tienen que ver con la motivación de la persona los factores de motivación que son el reconocimiento por la labor que realiza, la responsabilidad por el trabajo ejecutado, el crecimiento profesional, todos estos contribuyen a la satisfacción por el trabajo que ejecuta.

El modelo de expectativas de Vroom (1964), el cual se basó en tres aspectos fundamentales: el primero referente a que el ser humano tiene un deseo interno por lograr sus objetivos que se traza, considerados como expectativas a futuro, el segundo aspecto se refiere como el individuo asocia su esfuerzo por su trabajo al lograr sus objetivos y por lo cual recibe una recompensa. Y el último aspecto es referente a la motivación que impulsan al individuo a considerarse como productivo al ser logrados sus objetivos este último es la consolidación de las expectativas como las recompensas.

\section{Conclusiones}

El liderazgo directivo si influye en la satisfacción laboral en los docentes de la Institución Educativa $\mathrm{N}^{\circ}$ 80074, Virú 2020, al ejecutarse una buena conducción institucional, con metas y objetivos claros, con un líder influyente, que inspira confianza, realiza actividades coordinadas y considera la labor de los docentes, siendo reconocido en un $95 \%$ excelente, también los docentes están de acuerdo con sus obligaciones y condiciones de trabajo en un $63 \%$ satisfactorio. 
La influencia del Liderazgo directivo en la Satisfacción laboral, según el coeficiente de determinación es de 51,4\% variabilidad de satisfacción laboral expresado por la influencia del Liderazgo directivo. El coeficiente de correlación de Pearson es de 0,717 alto grado de correlación positiva entre la variable independiente y la dependiente.

La influencia del Liderazgo directivo en la Significación de la tarea, según el coeficiente de determinación es de 7,3\% la variabilidad de Significación de la tarea de la satisfacción laboral, expresada por la influencia del Liderazgo directivo. El coeficiente de correlación de Pearson es 0,270 grado de correlación positiva baja entre la variable y la dimensión.

La influencia del Liderazgo directivo en Condiciones de trabajo, según el coeficiente de determinación es 44,2\% la variabilidad de Condiciones de trabajo de la satisfacción laboral esta expresada por la influencia del Liderazgo directivo. Y el coeficiente de correlación de Pearson es 0,665 grado de correlación positiva moderada, entre la variable y la dimensión.

La influencia del Liderazgo directivo en Beneficios económicos según el coeficiente de determinación es 8,9\% la variabilidad de Beneficios económicos de la satisfacción laboral esta expresada por la influencia del Liderazgo directivo. El coeficiente de correlación de Pearson es 0,299 existe un grado de correlación positiva baja entre la variable y la dimensión.

\section{Referencias Bibliográficas}

Anaya, D., y Suárez, J. M. (2010). Satisfacción laboral de los profesores de Educación Infantil, Primaria y Secundaria: Un estudio de ámbito nacional. Revista de Educación, 344, $217-$ 243. https://doi.org/10.4438/1988-592X-RE-2014-365-266

Ander-Egg, E. (1997). Diccionario de Pedagogía. Buenos Aires. Argentina: Magisterio

Bernal. A., J. L. (2000). Liderar el cambio: El liderazgo transformacional. Lima. Perú.

Bolívar B., A. (agosto, 2010). ¿Cómo un liderazgo pedagógico y distribuido mejora los logros académicos? Revisión de la investigación y propuesta. Magis. Revista Internacional de $\begin{array}{llll}\text { Investigación en } & \text { Educación, } & \text { 3(5), }\end{array}$ https://www.redalyc.org/articulo.oa?id=281023476005

Cantón M., I.; y Téllez M., S. (2016). La satisfacción laboral y profesional de los profesores. Revista Lasallista de Investigación, 13(1). 214-226.

Cavalcante, J. (2004). Satisfacción en el trabajo de los directores de escuelas secundarias públicas de la región de Jacobina (Bahía-Brasil). [Tesis pregrado, Universidad Autónoma de Barcelona, http://www.tdx.cat/bitstream/handle/10803/5042/jjcs1de1.pdf?sequence=1

España].

Chiavenato I. (2009). Gestión del Talento Humano. Tercera edición. México: McGrawHill.

Cigna International Markets Observatorio de recursos humanos. (junio, 2020) La satisfacción laboral de los empleados mejora desde el confinamiento. https://www.observatoriorh.com/orh-posts/la-satisfaccion-laboral-de-los-empleadosmejora-desde-el-confinamiento.html

Cloninger S. C. (2002). Teorías de la personalidad. Tercera edición. México: Pearson.

Collao, O. (1997). Administración y gestión educativa. Lima. Perú: Asociación Gráfica Educativa

Esta obra se comparte bajo la licencia Creative Common Atribución-No Comercial 4.0 International (CC BY-NC 4.0) 
Cuya G., L. A. (2020). Liderazgo del director y satisfacción académica de los oficiales alumnos del diplomado en Liderazgo y Gestión del Grupo de Artillería, de la Escuela de Artillería del Ejército - 2018. [Tesis doctoral. Universidad Nacional de educación Enrique Guzmán y Valle]. http://repositorio.une.edu.pe/handle/UNE/4491

Días, E. (2015). Nivel de satisfacción laboral de los profesores de inglés de la Institución Educativa Privada Santa Margarita de Surco, Lima - Perú. [Tesis de Maestría en Educación con Mención en Teorías y Práctica Educativa. Universidad de Piura. Facultad de Ciencias de la Educación]. Piura, Perú. (4), 209-214

Fischman, D. (2000). El espejo del líder. Lima-Perú: UPC El Comercio.

Garay O. S. (2016). Liderazgo y logros en las organizaciones escolares de Chile. [Tesis Doctoral. Universidad Complutense De Madrid. Madrid]. España. https://eprints.ucm.es/40417/

Gibson, J., Ivancevich, J. y Dunnelly, J. (1993). Organizaciones. México: McGraw-Hill.

Gil V., F. (2014). Instituto de Capacitación Política. México D.F: Instituto de Capacitación Política.

Gutiérrez. H. (2003). El liderazgo Transformacional en el Docente Universitario. Panamá

Hannoun, G (2011). Satisfacción Laboral. [Universidad Nacional de Cuyo Facultad de Ciencias Económicas]. Mendoza - Argentina.

Hernández, R., Fernández, C. y Baptista, P. (2010). Metodología de la Investigación. $5^{\text {ta }}$ Ed. México, D.F., México: McGraw Hill Interamericana.

Herzberg, F., Mausner, B. y Snyderman, B. (1967). The motivation to work. (2 $\left.2^{\mathrm{a}} \mathrm{ed}.\right)$. New York: Wiley.

Inga, W. (2016). El clima organizacional y su relación con la satisfacción laboral en los docentes de la institución educativa primaria emblemática $N^{\circ} 70010$ gran unidad escolar San Carlos de la ciudad de Puno, 2015.

Leithwood, K. (1994). Liderazgo para la reestructuración de las escuelas. Revista de Educación, 304, 31-60.

Lidstone J. (2009). La Motivación del equipo de Ventas. Ediciones Deusto. España.

Longo, M., F. (2008). Liderazgo distribuido, un elemento crítico para promover la innovación. http://docentesinnovadores.perueduca.pe/wpcontent/uploads/2017/03/larticuloliderazgodistribuido.pdf

Machaca M., R. G. (2019). Gestión Administrativa, Liderazgo Directivo y Satisfacción Laboral en Docentes de las Instituciones Secundarias Públicas de la Ciudad de Puno. [Tesis doctoral. Universidad Nacional de educación Enrique Guzmán y Valle] http://repositorio.une.edu.pe/handle/UNE/4101?show=full

Ministerio de educación (2014). Marco del buen desempeño directivo. Directivos construyendo escuela. Lima-Perú

Manosalvas Vaca, Carlos Anibal, y Manosalvas Vaca, Luis Oswaldo, y Nieves Quintero, Jorge (2015). El clima organizacional y la satisfacción laboral: un análisis cuantitativo riguroso de su relación. AD-minister, (26), 5-15. ISSN: 1692-0279. https://www.redalyc.org/articulo.oa?id=3223/322339789001

Montañez A., E. J. (2019). Liderazgo directivo, motivación y desempeño docente en las instituciones educativas del nivel secundario del distrito de Platería-Puno. [Tesis doctoral. Universidad Nacional de educación Enrique Guzmán y Valle] http://repositorio.une.edu.pe/handle/UNE/4139?show=full

Maslow, A. H. (2005). El Management Según Maslow: Una Visión Humanista para la Empresa de Hoy. Barcelona: Paidós Ibérica. 
Muñoz-Secoa, E., Coll-Benejam, J., Torrent-Quetglas, M. y Linares-Pou, L. (2006). Influencia del clima laboral en la satisfacción de los profesionales sanitarios. Atención Primaria.

Palma, C. Sonia. (2006). Escala de Satisfacción Laboral (SL-SPC) Manual (Editora y Comercializadora CARTOLAN EIRL.), Perú.

Tovar V., M. R. (2018). Influencia del liderazgo del director en la satisfacción del alumno del diplomado en liderazgo y gestión del Grupo de Artillería de la Escuela de Artillería del Ejército - 2017. [Tesis doctoral. Universidad Nacional de educación Enrique Guzmán y Valle]. http://repositorio.une.edu.pe/handle/UNE/4031

Robbins, S. P. (2006). Diagnostico Organizacional. 13 Edición Editorial. México: MC Graw Hill.

Salazar Ch., M. y Pinedo M., M. (2011). Relación del clima institucional con el desempeño docente en la Institución Educativa Angélica Palma Román Lima - 2011. http://es.scribd.com/doc/55416611/27/EL-LIDERAZGODIRECTIVO-EN-LAINSTITUCION

Stoner. J. Freeman R. Y cols (1996) Administración. México. Ed. Pearson.

Thieme J., C. P. (2005). Liderazgo y eficiencia en la Educación Primaria - El caso de Chile. http://tdx.cat/handle/10803/3958

Urquijo, J., y Bonilla, J. (2008). La remuneración del trabajo, manual para la gestión de sueldos y salarios. Caracas-Venezuela: Universidad Católica San Andrés Bello.

Vroom, V. H. (1964). Work and Motivation. $2^{\mathrm{a}}$ Edición. Nueva York: Wiley and Sons. 